\title{
Sustainable Urban and Transport Development for Transportation Disadvantaged: A Review
}

\author{
Tan Yigitcanlar ${ }^{*}$, Kushairi Rashid and Fatih Dur
}

\author{
Queensland University of Technology, School of Urban Development, Brisbane, Australia
}

\begin{abstract}
Around the world, particularly in North America and Australia, urban sprawl combined with low density suburban development has caused serious accessibility and mobility problems, especially for those who do not own a motor vehicle or have access to public transportation services. Sustainable urban and transportation development is seen crucial in solving transportation disadvantage problems in urban settlements. However, current urban and transportation models have not been adequately addressed unsustainable urban transportation problems that transportation disadvantaged groups overwhelmingly encounter, and the negative impacts on the disadvantaged have not been effectively considered. Transportation disadvantaged is a multi-dimensional problem that combines demographic, spatial and transportation service dimensions. Nevertheless, most transportation models focusing on transportation disadvantage only employ demographic and transportation service dimensions and do not take spatial dimension into account. This paper aims to investigate the link between sustainable urban and transportation development and spatial dimension of the transportation disadvantage problem. The paper, for that purpose, provides a thorough review of the literature and identifies a set of urban, development and policy characteristics to define spatial dimension of the transportation disadvantage problem. This paper presents an overview of these urban, development and policy characteristics that have significant relationships with sustainable urban and transportation development and travel inability, which are also useful in determining transportation disadvantaged populations.
\end{abstract}

Keywords: Transportation disadvantaged, social exclusion, sustainable transportation, sustainable urban and transportation development, travel demand management, accessibility, mobility.

\section{INTRODUCTION}

The concept of sustainability and its applicability to urban settings have been one of the most discussed issues in the literature [1]. As rapid urbanisation and growing population of cities are considered, implications of changing lifestyles on sustainability and how these are remedied could be considered as the most pressing subject of the urban and transportation planning professions. The complex nature of cities and politics around them strongly force urban and transportation planners to analyse contemporary problems of their cities more carefully. This also pushes them to produce more effective policy recommendations. In this instance, the popular concept of 'sustainable urban and transportation development' (SUTD) comes into play. The most vulnerable groups affected from the unsustainable urban and transportation development are referred as 'transportation disadvantaged' (TDA) groups. TDA groups are generally identified as those people whose range of travel alternatives are limited, especially limited to the availability of easy-touse and inexpensive options of trip-making [2]. The negative impacts of transportation on TDA have not been effectively considered in the urban and transportation modelling studies, as these models do not take all of the demographic, spatial and transportation service parameters into account [3]. Particularly land-use and transportation models are

*Address correspondence to this author at the Queensland University of Technology, School of Urban Development, Brisbane, Australia; Tel: +61.7.3138.2418; Fax: +61.7.3138.1170;

E-mail: tan.yigitcanlar@qut.edu.au increasingly under attack for being biased against nonmotorised traffic modes and TDA groups [4], and for failing to inform policy-makers with accurate information on TDA. In recent years, a strong demand has arisen for an equitable access to transportation opportunities for TDA. The literature points out the ethical responsibility of modelling studies towards social issues and views 'accessibility' and 'social equity' among the key issues for urban and transportation planning $[5,6]$.

Determining TDA populations and comparing their characteristics with non-disadvantaged groups are important for a sound urban and transportation policy-making and achieving SUTD. One of the major deficiencies is the unavailability of a comprehensive and holistic approach in determining TDA and measuring their disadvantage levels. Existing land-use and transportation planning models do not provide policy-makers with the degree of disadvantage levels of a locality, and have been inadequate in addressing severe long-term transportation problems that TDA groups overwhelmingly encounter [7-9]. Policy-makers would be able to propose relevant remedies or polices, if they have accurate disadvantage ratios and indicators.

The aim of this paper is to provide a review and outlook on the key features of SUTD and TDA by linking literatures on both of these fields. The two sections following this introduction provide a thorough review of the literature on SUTD and TDA. The fourth section of the paper firstly reveals the key characteristics of TDA by introducing a conceptual framework that accommodates the key indicators of measuring and determining TDA. Secondly it presents an 
overview of these urban, development and policy characteristics that have significant relationships with SUTD and travel inability, which are also useful in determining TDA populations. The paper concludes by pinpointing the key findings and also directions for further research in the intersecting areas of SUTD and TDA.

\section{SUSTAINABLE URBAN AND TRANSPORTATION DEVELOPMENT}

Economic, environmental and social dimensions of SUTD encompass urban form, transport, infrastructure and community domains. These domains also identify the responsibilities of planning in terms of intervention and regulation to reach desired sustainable urban futures. Particularly, critical importance of the problems related to unsustainable transportation activities, such as greenhouse gas emissions, health-threatening pollutants, non-renewable fuel use, traffic congestion, accidents and fatalities, have revealed the need for SUTD. On the one hand, mostly at the global and national levels, transportation emissions and energy consumption issues have been heavily discussed and received a great deal of attention. Following this attention, at the technological level, improvement strategies in the fuel and engine systems of vehicles have become one of the priority areas of development. On the other hand, policies aiming to diminish motor vehicle dependency and excessive travel have been also taken into account for achieving the long term sustainability goals of many urban areas. In the literature these policies are grouped under the heading of 'travel demand management' (TDM) considerations. As technological advancement of vehicle systems and transportation infrastructure constitute mostly technical and engineering part of the discussion, TDM mainly focuses on relationship between urban form and transportation, and socio-economic dimensions, such as household characteristics affecting location choice, daily and holiday travels, mode choice and acceptability of TDM policies. Supporters of TDM discuss the relative importance of topdown approaches, which assign the main SUTD responsibilities to local governments and planning agencies [10]. However in more recent studies, it is reported that bottom-up strategies, which initiate public awareness towards individuals' contribution to unsustainable transportation pattern and searching the ways of voluntary travel behaviour change, may be more effective than topdown approaches [11].

Inherent complexity of achieving SUTD is mainly explained as a consequence of a number of factors influencing urban form and transportation, joint effects of this relationship, and time-dependent changes in urban structure. Land-use decisions affect transportation investments and systems in the short run, however, travel pattern shaped according to transportation network affects land-use decisions and future transportation systems in the long run [12]. In SUTD, urban form discussion involves mainly density and mix land-use dimensions acknowledging the urban sprawl problem. Furthermore, motor vehicle dependency and urban sprawl relationship is another popular topic of SUTD [13]. Principal function of urban consolidation via intensification and mix use reduces trip lengths and total travel, and also changes modal split from automobile dependence to more sustainable public transportation and non-motorised means [14].

In terms of social equity and accessibility to urban services, low density urban sprawl imposes economic and social burdens on low income groups towards deterioration of community sense and feeling powerless [15]. It is also asserted that neo-traditional settlement form satisfying high density and mix use features are more sustainable that suburban type urban development [16]. Conceptualisation of SUTD policies has revealed various urban planning movements, such as smart growth, new urbanism, transit oriented development, and decentralised concentration. Opponents of urban consolidation, put forward the questions of feasibility and acceptability of such policies. For example, it is claimed that high density and large investment to public transportation may not warrant a reduction in motor vehicle travel due to high level motor vehicle travel preferences of the people [17]. Another claim opposing to compact urban form states that neo-traditional urban form, like in Europe, does not necessarily make people less motor vehicle dependent, what forces people to travel less is high public transportation patronage, high fuel prices and stringent tax policies [18]. From the perspective of land market economics, urban containment and consolidation could result in an increase in land and property values, which unfortunately strengthens the suburbanisation trend that stimulates motor vehicle mobility in return [15].

In the literature, SUTD arguments frame the main considerations demanding change towards a more sustainable state. However, the causal relationship among various factors affecting urban form and transportation interrelationship is the main subject of the TDM domain. Interestingly, growing common interest on social exclusion, public health and transportation relationship, physical activity, and non-motorised transportation mode preference leads to the convergence of three literature: social exclusion, travel behaviour and physical activity research $[1,19,20]$. The common arguments of these disciplines are opportunities, patterns and preferences of travel, mode choice, socio-economic drivers of motor vehicle ownership and travel, and effects of urban design consideration on physical activities, especially walking and cycling [21].

The balance between mobility and accessibility plays a key role in achieving SUTD. Mobility impairment and low level of accessibility to urban services and transportation facilities are among the growing problems contributing to the escalation of transportation inequity $[22,23]$. Particularly in the context of North America and Australia, distance to services is increased over the last three decades together with the rapid growth of suburbia. There is a clear inequity problem between people with and without an automobile, and those without access to an automobile are even deprived of access to the economic and social life of the city [24]. In the light of the mobility and accessibility discussions, two key questions have become evident from the recent literature. The first one is whether the new direction of SUTD policies is focused only on mobility and technology issues. The second question is whether SUTD policies also consider citizens' accessibility to urban opportunities, particularly for those who are TDA [25]. This second question aligns with the social dimension of sustainability, 
whereby sustainability policies should take into account of social considerations together with environmental and economic sustainability issues [26]. It also relates to the arguments about efficiency versus equity of market and governmental regulations. These arguments suggest that policies that increase the cost of travel should not be the only regulatory instruments, but that other compensating measures, such as decreasing labour tax, which covers the increased transportation cost of low-income people [27]; and subsidising public transportation and other low cost transportation infrastructure and services, particularly in the areas where transportation disadvantages exists [28]. Some contend that such measures would rectify the erroneous definition of sustainable transportation, which equates mobility to accessibility, by emphasising the accessibility to public services and urban activities with various transportation means [23, 29]. Transportation policies, while improving people's mobility, should also convey social equity in sustainability. Further, a sustainable and socially just transportation system ought to provide a fair distribution of transportation services and equal access to employment, housing, education, health services and recreation, and minimise the level of TDA [30].

\section{TRANSPORTATION DISADVANTAGED}

Many cities, particularly in North America and Australia, are plagued by the motor vehicle-oriented suburbanisation, which is a development characterised by low-density sprawl like development, big retailers replacing corner shops, doubled-up distances to major local activities, and removal of public transportation lines from the poor districts [24]. In such development segregated view of the urban space can even have a larger responsibility in the resulting appearance of the disadvantage. Poor local transportation system has a role in creating barriers for TDA or also so-called 'socially excluded' groups that have become more and more inaccessible [48]. Combination of poor accessibility with low levels of mobility and low levels of sociability intensifies TDA [6]. In such circumstances what really matter is to provide more assistance to the most vulnerable groups, poor, elderly and disabled particularly in rapidly aging societies [30].

A number of studies have been conducted in order to determine TDA population. For example, Duvarci and Yigitcanlar's study [30] sought integration of TDA analysis with mainstream travel demand models by employing perceptional data, in which TDA could neatly be determined through a cluster analysis focusing on community travel conditions in Aydin, Turkey. Some UK-based studies used accessibility measures to determine TDA [19]. For instance, Schmocker et al. [31] determined the trip making characteristics of elderly and disabled for four trip purposes by analysing the 2001 London Area Travel Survey. Wu and Hine [22] provided a classification on TDA by deprivation domains of income, employment, health and disability, education, geographical access to services, social environment, and housing. Church et al. [19] defined seven basic TDA types as: physical, geographical, exclusion from facilities, economic, and time-based, fear-based and spacebased exclusions. Despite the growing literature and interest on the recent call of governmental policy on social exclusion in the UK, there are still both methodological and conceptual struggles in tackling the TDA issue comprehensively.

Not all widely accepted parameters to measure TDA could be used under every circumstance. For instance, sometimes TDA may spend less money and time for their trips due to less mobility. Likewise, travel is assumed to be an impediment, which is true especially for work trips, a cost to be reduced whenever possible [32]. Similarly increasing leisure activities and the time allowed to such trips may not be assumed costly, thus, the accurate modelling of leisure trips becomes more critical especially for TDA groups. Thus, it is useful if TDA groups are determined for each trip purposes. Also inequity arises between people who have and who have not own or access to a motor vehicle. Yet, the cost of driving in some developing countries is quite high due to higher fuel prices and ever-increasing congestions, thus owning a motor vehicle does not make them nondisadvantaged, which is not the case in North America or Australia, where driving is more affordable due to lower fuel prices and affordable motor vehicles. Additionally, peak hour congestion is many times perceived as the biggest problem, but the perception is relative and endurance to the congestion changes from one culture to another and from metropolitan areas to remote settlements. Income levels also may have different implications on difficulty perceptions. Moreover, even disabled or elderly may feel nondisadvantaged, if they are provided with easy accessibility and mobility options.

TDA is a dynamic and multi-dimensional issue involving physical, temporal, economical, spatial, and psychological dimensions [33]. Because of the multi-dimensional nature of TDA serious measurement and level of analysis difficulties arise [6]. Most of the related research, while managing to locate the problem and enriching discussion and convergence, failed to determine TDA accurately. Because of the cultural significance of the issue, travel behaviours may show variety in different cultures and even from one disadvantaged group to another [34]. Thus, determination of TDA can be highly place, culture and context dependent. Determining TDA populations and comparing their characteristics with non-disadvantaged groups are extremely important for a SUTD and urban policy-making. One of the major deficiencies is the unavailability of a comprehensive and holistic way in determining TDA groups and measuring their disadvantage levels. Existing transportation planning models do not provide policy-makers with the degree of disadvantage levels of a locality, and have been inadequate in addressing severe long-term transportation problems that TDA groups overwhelmingly encounter [7, 8]. Policymakers would only be able to propose relevant remedies or polices, if they have accurate disadvantage ratios and indicators. Therefore, it is important to clearly understand the specific characteristics of TDA.

\section{CHARACTERISTICS OF TRANSPORTATION DIS- ADVANTAGED}

Transportation disadvantage is relatively a function of accessibility and mobility problems. In general, both accessibility and mobility can be conceived as an ease to reach a destination [35]. Both terms have been fairly used together in the TDA discourse. There are many factors or 
indicators that are used for determining accessibility and mobility levels of individuals and social groups, such as social, economic, transport and land-use indicators.

In the discourse of equity in transportation supply, mobility level has been used substantively to identify social groups that have lower mobility levels and have significantly higher needs and demands for travel [10]. There is certainly a close relationship between access to a motor vehicle or income levels with the ability to travel freely [36]. It is identified that a social group with lower mobility level is traditionally lack one of these two resources. Therefore, the elderly, children and youth, caretaker, low income group, beneficiaries, unemployed, women and those who physically impaired are traditionally associated with individuals or social groups that are TDA [37]. However, transportation disadvantage does not solely depend on the demographics or socio-economic attributes of people (e.g. income), but also is an outcome of the transportation services (e.g. public transportation) and spatial (e.g. density) characteristics of the locality.

SUTD, including provision of efficient public transportation systems, can resolve TDA and accessibility problems in a society by providing services those without any access to motor vehicle alternatives. On the one hand, it is vitally important that sustainable public transportation systems to provide the community with a significant level of travel opportunity with minimum travel time, distance and cost to essential destinations. On the other, it is important that sustainable public transportation system to provide a reasonable access from origin to public transportation stop or station by providing a high public transportation coverage area with a well connected bicycling and pedestrian system [4]. However, TDA is not merely caused solely by the aforementioned factors, but it also includes preferences that are perceived by the passengers. In this regard, an individual maybe considered as disadvantaged based on the merits of the public transportation vehicles' comfort, waiting time and interchange locations and above all service frequency, reliability and punctuality [27]. Furthermore; there are other factors that have significant weighting in determining transportation disadvantage such as the safety level in stations or stops, customer care and travel information [2]. Moreover, those who are disable, with a big luggage or travelling with children could be considered disadvantaged while travelling via public transportation. These groups find that access to and from a bus, train and station are sometimes troublesome due to unfriendly design of the system and the environment.

TDA can be a result of spatial characteristics or location disadvantage. In this case, the location disadvantage is partly due to mono-centric development and the dramatic growth of a city that lead to urban sprawl and low density suburban expansion [35, 38]. In mono-centric development form, the fast phase of the spatial development is unbearable for transportation infrastructure to cope and thus, the low residential development is unprofitable for the public transportation operators. Furthermore, location disadvantage also is a result of the increasing land values in the inner city. As land value increases in the inner area of the CBD, the low income groups have to move from the inner city and settle in the outer areas which in general have a relatively low mobility level and a high distance from the urban centre and many key land-use destinations. Despite that the condition could be worsening as the low density development in the suburbia is invisible for facilities and amenities to operate and consequently, these households have to bare the increased of travel cost due to increase in travel distance. While the current land-use and infrastructure policy remains encouraging motor vehicle ownership, providing limited public transportation services in the suburbia, and creating a severely disadvantaged situation for these groups.

To remedy TDA problems, Banister [7] suggests equilibrium to be developed between all elements of landuse and transportation planning that considers both physical (e.g. urban form and traffic) and social dimensions of transportation (e.g. people and proximity). The sustainable mobility approach requires actions to reduce the need to travel fewer trips, encourage modal shift, reduce trips lengths and encourage greater efficiency in the transportation system. Therefore, there exists a substantial need to identify which spatial characteristics most leads to a severe TDA. Even though, socio-economic characteristics and public transportation system indices are also have significant roles in determining TDA, it has been identified that only a few TDA research include detailed spatial characteristics in their analysis.

TDA has a multi-dimensional nature that consists of demographic, spatial and transportation service dimensions. Fig. (1) developed as part of the research reported in this paper, illustrates key dimensions and characteristics with their literature references in determining TDA. The literature indicates that there is a fundamental difference between the travel needs of the people and their travel behaviours. Thus, measuring the difference between these two might help planners and policy-makers to determine TDA and develop policies to overcome their disadvantages. Therefore, it is vital to clearly scrutinise both the travel needs and also behaviours of populations. This brings the importance of assessing both travel demand and also supply. In broad terms, the difference between supply and demand indicates what needs to be done by policy-makers and planners. The conceptual framework, shown in Fig. (1), focuses on TDA determination by both measuring travel demand and supply. In this framework in terms of travel demand key socioeconomic characteristics that effect accessibility and mobility levels are considered. For the travel supply side public transportation infrastructure and service characteristics are seen as the main elements. Between the two dimensions, demographic and service, the framework places the spatial dimension domain. Urban, development and policy characteristics of this spatial dimension of a locality is also important in determining TDA populations in that particular locality.

Although, there are three equally important, demographic, spatial and service, domains or dimensions of TDA determination, a substantial number of research mainly have focused on the analysis of the socio-economic and public transportation service characteristics of TDA. There have been relatively limited explorations on the spatial dimensions and its urban, development and policy characteristics. The reason of why spatial dimension of TDA has not been scrutinised as much as the others might most 


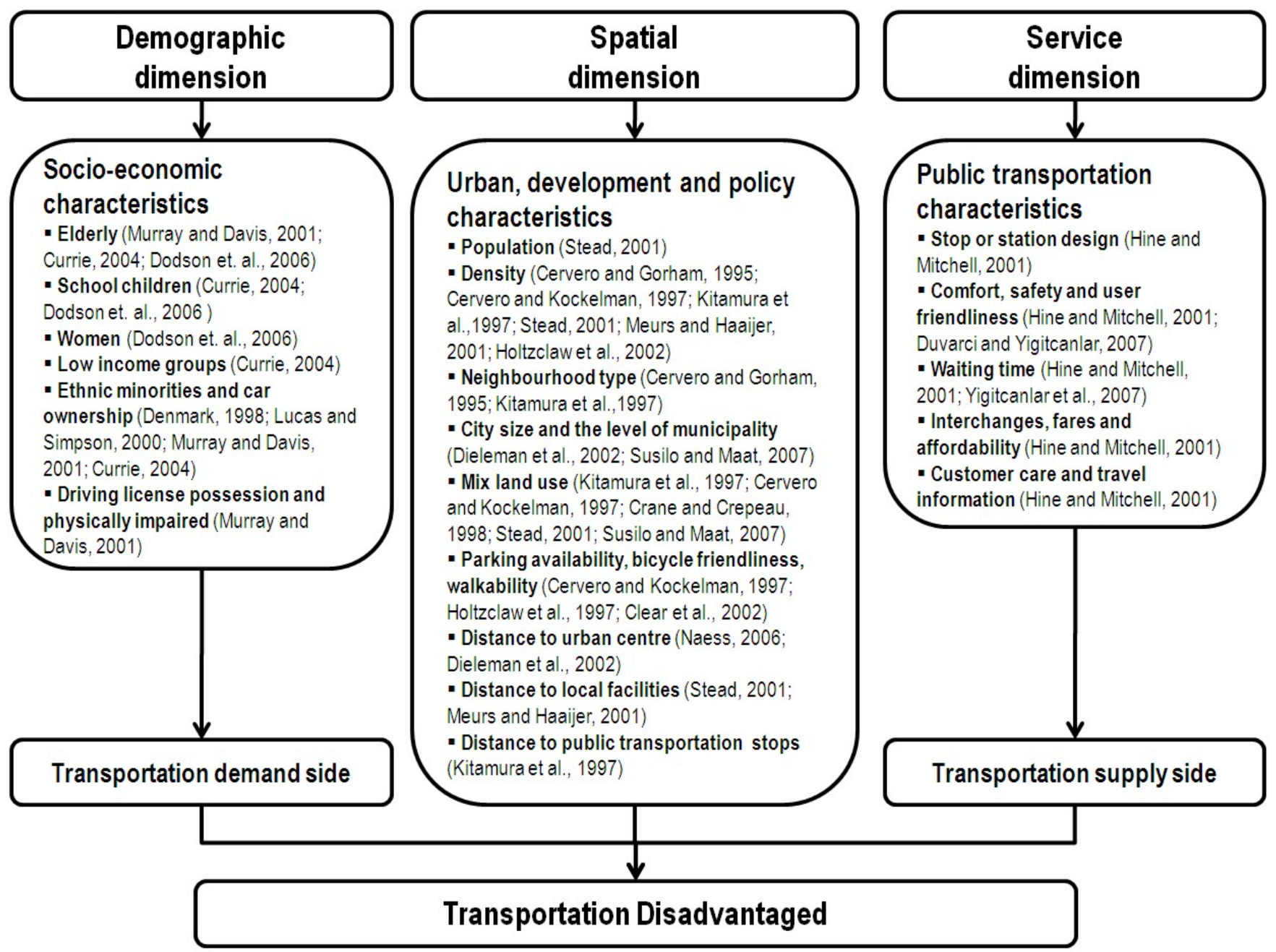

Fig. (1). A conceptual framework for transportation disadvantage analysis.

probably be due to the complex process of quantifying TDA and finding the most suitable indicators for analysis. Therefore, this paper mainly explores this relatively less investigated dimension of TDA.

From the practice of TDA determination exercises, it is noticeable that spatial indices of TDA are determined by using two distinctive approaches. The first approach is concerned of trip making by which trip capabilities are depicted by trip rates, trip frequencies or trip distances or vehicles kilometres travelled. It is acknowledged that, the ease to travel is likely associated with high level of trip rates and trip frequencies, which is to say TDA can be clearly defined with longer travel distance, increased dependency to private vehicle use, low trip rates and low trip frequency [24]. The second approach is the accessibility-based measures that identify TDA by using accessibility levels or number of accessible land-use destinations [6]. In this measurement approach, people may become TDA, if the available transportation infrastructure does not reach to the desired destination, especially for the mandatory trips of education, health and work. Determining TDA by using this approach requires expanding the data input with more information of urban, development and policy characteristics [24]. In this approach vital land-use datasets, such as location of activities, distribution of services, and the link between residential, determine the accessibility of an area and thus determine the level of TDA.

Keeping above mentioned two TDA approaches in mind the following section investigates and provides a review of the major spatial measures used for determining TDA.

\section{SPATIAL MEASURES FOR DETERMINING TRANSPORTATION DISADVANTAGED}

High dependence on motor vehicle use for travel has been clearly associated with unsustainable way of travel due to the relatively high cost of travel and environmental hazards that motor vehicle mobility causes. Relating SUTD with TDA, the literature underlines certain type of built environment and land-use characteristics that determine travel behaviours, particularly mode choice, motor vehicle travel distance, and degree of motor vehicle dependency [24]. Spatial planning is vital in providing sustainable transportation systems and sustainable mobility as it determines the spatial arrangements of land-uses and amenities. One of the SUTD strategies sensitive to TDA is developing an inclusive urban form with high mobility opportunities by delaminating physical separation between activities, reducing distance between activity centres and by promoting significant level of mix land-use development. Longer motor vehicle travel, high level of motor vehicle 
dependency, and low accessibility are significantly associated with land-use characteristics such density of the development, distance from urban centre, mix land-uses, type of neighbourhood, and presence of pedestrian facilities [24]. The literature on spatial measures, travel capabilities and TDA are generally determined based on trip distance, trip frequency, and mode of travel. These indicators, factors or variables along with other supporting ones are used interchangeably to show travel capabilities and indicating TDA.

The key spatial factors that affect travel capabilities of TDA include: urban density, urban form, urban structure, settlement size, distance to urban centre, distance to local facilities, land-use mix, neighbourhood type and street design. The key characteristics of these factors are discussed below.

\section{Urban Density, Form and Structure}

Density is the most studied land-use characteristic that has the largest share to explain numerous travel behaviours, and achieving high density is a SUTD goal [39]. In general, density determines travel behaviours for four reasons. Firstly, higher densities increase the range of opportunities for the development of local personal contacts and activities that can be maintained without resort for motorised travel. Secondly, higher population densities broaden the range of local services thus reduce the need of travel and length of travel. Thirdly, higher density tends to reduce travel distance between homes, services, employment and other opportunities which reduces travel distance. Fourthly, higher density provides substantial number of passengers for public transportation services and less amenable to private motor vehicle usage. Therefore, there is significant ground to ascertain that people residing in less dense areas have a higher possibility to become TDA. Findings of Stead's [40] study show that residents of larger and dense urban areas travel less than residents of smaller and spread population settlements. Thus, it would not be wrong to say low density suburbs travel longer distances than the residents of higher density suburbs. In relation with mode choice, density also has a considerable influence in distance travelled by private motor vehicles. A study on U.S. cities by Kitamura et al. [41] shows that density is the most significant land-use characteristic in mode choice and TDA. This finding is also supported by other studies undertaken by Holtzclaw et al. [42] and Meurs and Haaijer [43].

\section{Settlement Size, Distance to Urban Centre and Local Facilities, and Land-Use Mix}

Job opportunities and centralised activities in urbanised areas attract substantial number of commuters, especially those residing in the suburban and outer areas. Compact urban form with services is at proximity in most urbanised areas have lead to shorter travel distance, thus increased the level of accessibility. Based on a research by Susilo and Maat [44], degrees of urbanisation variables consistently influence the parameter of commuting journey whereby travel distance has a negative correlation with the size of the settlement. Dieleman et al. [45] reveal that motor vehicle use is likely to be low in the large and medium size cities and high in suburban or rural areas. As the distance from an urban centre increases, people who reside in the outer areas become more dependent to motor vehicle use and have a higher distance to travel by private motor vehicles. According to Naess [46], travel capability to the urban centre has a negative correlation with distance from the urban centre. As the travel distance from urban centre increases, trip frequency decreases and participation in urban activities also decreases. The distant area from urban centre is more reliable on service in the local centre and the absence of such services in the neighbourhood may increase the motor vehicle use. In the perspective of SUTD, the increased distance from urban centre has a negative consequence to energy consumption and level of sustainability. According to Holden [1], the increased distance from urban centre has a positive correlation with energy consumption, therefore, in order to promote a lower energy consumption a compact development with minimised travel distance is favourable. This also applies for TDA populations, as the distance from urban centre increases the level of TDA shows an increase [30].

The provision of facilities and services within residential areas is a significant land-use characteristic that can reduce travel distance and increased travel capabilities via nonmotorised vehicles. The level of mix land-use is commonly identified by using a ratio of job and housing balance. However, mix land-use can also be identified by using other proxy variables that depict mix land-use characteristics such as provision of facilities and distance to local facilities. Litman and Burwell [10] reveal that in order to develop a good merit in accessibility, residents of an area should have right to access to emergency services, healthcare, basic food and clothing, education and employment, public services, mail and freight distribution, and social and recreational activities. The presence of facilities within a residential area contributes to lower motor vehicle mobility, more travel capabilities and less TDA populations. Meurs and Haaijer [43] and Stead [40] indicate that travel capabilities can be increased considerably by locating closely daily facilities, shopping and school with close proximity to residential areas. The results of Cervero and Kockelman's [34] research on travel behaviours and mix uses show that residential areas with modest or high spatial accessibility to commercial premises tend to have less vehicles kilometres travelled per household. Thus, large share of commercial areas in residential zones allows a high level of travel capability to its residents, via public or non-motorised modes, and contributes to the minimisation of TDA within the locality [47]. Stead [40] claims that higher job ratio is clearly associated with low travel distance. Examining the influence of settlements and commuter characteristics, Susilo and Maat [44] identify that a high ratio between job and house tend to reduces journey outside the neighbourhood.

\section{Neighbourhood Type and Street Design}

The influence of neighbourhood type and street design on the trip making and travel behaviours have been debated considerably [14]. It can be said that certain land-use features have noticeable influence on travel behaviours and trip making. Within the scope of neighbourhood type and street design studies, two noticeable features have been subject of investigation. The first one is transit oriented development and neighbourhoods that the physical form of this type of neighbourhoods favour pedestrian and public 
transportation use, and have gridiron street lay-out. These types of neighbourhoods are labelled as green transportation friendly neighbourhoods that help lowering down the TDA numbers. Cervero and Groham [48] state that in the U.S. most public transportation neighbourhoods were largely built before 1945, and auto-oriented neighbourhoods, also known as suburban neighbourhoods, are relatively new neighbourhoods developed without having any considerable notion on the public transportation use [41]. This type of development is generally built in an area without public transportation lines and primarily has random street patterns with having a combination of: 3-way lanes, T-intersections, and cul-de-sacs. Cervero and Groham's [48] study compares commuting characteristics of transit oriented neighbourhoods with auto-oriented neighbourhoods. The findings of this study show that public transportation neighbourhood has a lower drive-alone modal share and trip generation rate than its auto-oriented counterpart. Public transportation neighbourhoods have higher walking and bicycling modal share than auto-oriented neighbourhoods. The presence of pedestrian friendly design features in the public transportation neighbourhood design has a significant contribution in increasing residential travel capabilities and SUTD [42]. A walkable neighbourhood combining necessary land-use destinations supported with a reliable public transportation minimises the private motor vehicle trips as well as the number of TDA dramatically $[41,49]$.

\section{CONCLUSION}

Past few decades have shown a significant increase in private motor vehicle ownership, over reliance on private motor vehicles for daily travel and an unsustainable urban development pattern. The over reliance, automobile dependency and unsustainable urban form correlate with a number of problems such as environmental degradation, accident-generated mortality, and increasing TDA levels. It has been widely accepted that current unsustainable practices and lifestyles cannot be sustained, if we are to leave a healthy living planet to the next generations. One of the most vulnerable groups affected from the unsustainable development is TDA, whose abilities and range of travel alternatives are limited. This circumstance urges for a need for a more sustainable way of planning urban and transportation development. In the light of these problems a new perspective, SUTD, has been pushed to the forefront of policy-making and politics. In this new sustainability perspective provision of transportation infrastructure and services that are sustainable is seen as a panacea for TDA.

The literature, reviewed in this paper, clearly indicates that discussions on incorporating sustainable development and transport disadvantage in an urban context are clustered around the broad topics of socio-economic, urban form and transportation features of localities as the key considerations. For that reason, this paper developed and presented a conceptual framework that illustrates the key dimensions for determining TDA as demographic, spatial and transportation service dimensions. Among these three dimensions the paper primarily investigated the spatial dimension of urban environments and introduced its urban, development and policy characteristics, which all are important in achieving SUTD and minimising TDA problems. However, our future research will also be focusing on the other two dimensions of
TDA, and the conceptual framework, presented in this paper, is planned to be further developed into an operational framework to form a TDA index to be used in determining TDA populations, measuring their disadvantage levels, and developing policy directions to inform local decisionmaking.

\section{REFERENCES}

[1] E. Holden, Achieving Sustainable Mobility: Everyday and LeisureTime Travel in the EU. London: Ashgate, 2007.

[2] J. Hine, and F. Mitchell, Transport Disadvantage and Social Exclusion. London: Ashgate, 2003.

[3] J. Hunt, D. Kriger, and E. Miller, "Current operational urban landuse-transport modelling frameworks: a review", Trans. Rev., vol. 25, no. 3, pp. 329-376, 2005.

[4] A. Murray, and R. Davis, "Equity in regional service provision", $J$. Reg. Sci., vol. 41, no. 4, pp. 557-600, 2001.

[5] E. Deakin, "Trends and policy choices", Access, vol. 23, no. 1, pp. 12-15, 2003.

[6] T. Yigitcanlar, N. Sipe, R. Evans, and M. Pitot, "A GIS-based landuse and public transport accessibility indexing model", Aust. Planner, vol. 44, no. 3, pp. 30-37, 2007.

[7] D. Banister, "Sustainable urban development and transport-a Eurovision for 2020", Trans. Rev., vol. 20, no. 1, pp. 113-130. 2000.

[8] S. Kenyon, G. Lyons, and J. Rafferty, "Transport and social exclusion: investigating the possibility of promoting inclusion through virtual mobility", J. Trans. Geogr., vol. 10, no. 3, pp. 207219, 2002.

[9] B. Simpson, Urban public transport today. London: E\&FN Spon, 1994.

[10] T. Litman, and D. Burwell, "Issues in sustainable transportation", Int. J. Glob. Environ. Issues, vol. 6, no. 4, pp. 331-347, 2006.

[11] W. Shore, "Land-use, transportation and sustainability", Technol. Soc., vol. 28, no. 1-2, pp. 27-43, 2006.

[12] P. Newman, "Sustainable transport for sustainable cities", Issues, vol. 1 , no. 1 , pp. 6-10, 2006.

[13] N. Low, B. Gleeson, and E. Rush, "Making believe: Institutional and discursive barriers to sustainable transport in two Australian cities", Int. Plann. Stud., vol., 8, no. 2, pp. 93-114, 2003.

[14] R. Ewing, and R. Cervero, "Travel and the built environment: a synthesis", Transp. Res. Record, vol. 1780, no. 1, pp. 87-114, 2001.

[15] E. Burton, "The compact city: just or just compact? A preliminary analysis", Urban Stud., vol. 37, no. 11, pp. 1969-1985, 2000.

[16] S. Handy, L. Weston, J. Song, and K. Lane, "Education of transportation planning professionals", Transp. Res. Record, vol. 1812, no. 1, pp. 151-160, 2002.

[17] P. Gordon, and H. Richardson, "Are compact cities a desirable planning goal?”, J. Am. Plann. Assoc., vol. 63, no. 1, pp. 95-106, 1997.

[18] M. Breheny, "The compact city and transport energy consumption", Trans. Inst. Br. Geogr., vol. 20, no. 1, pp. 81-101, 1995.

[19] A. Church, M. Frost, and K. Sullivan, "Transport and social exclusion in London", Trans. Policy, vol. 7, no. 3, pp. 195-205, 2000.

[20] B. Giles-Corti, "People or places: What should be the target?", $J$ Sci. Med. Sport, vol. 9, no. 5, pp. 357-366, 2006.

[21] T. Yigitcanlar, and F. Dur, "Developing a sustainability assessment model: the sustainable infrastructure land-use environment and transport model", Sustainability, vol. 2, no. 1, pp. 321-340, 2010.

[22] B. Wu, and J. Hine, "A PTAL approach to measuring changes in bus service accessibility”, Trans. Policy, vol. 10, no. 1, pp.307-320, 2003.

[23] T. Yigitcanlar, L. Fabian, and E. Coiacetto, "Challenges to urban transport sustainability and smart transport in a tourist city", Open Transp. J., vol. 2, no. 1, pp. 19-36, 2008.

[24] K. Rashid, and T. Yigitcanlar, and J. Bunker, "Transportation disadvantage determination framework: space-time organisation measure in determining transportation disadvantage", The 2nd Infrastructure Research Theme Postgraduate Student Conference, pp. 260-272, 2009.

[25] P. Moriarty, "Environmental sustainability of large Australian cities", Urban Policy. Res., vol. 20, no. 3, pp. 233-244, 2002. 
[26] M. Roseland, "Sustainable community development: integrating environmental, economic, and social objectives", Progr. Plann., vol. 54, no. 2, pp. 73-132, 2000.

[27] N. Cass, E. Shove, and J. Urry, "Social exclusion, mobility and access", Sociol. Rev., vol. 53, no. 3, pp. 539-555, 2005.

[28] J. Black, A. Paez, and P. Suthanaya, "Sustainable urban transportation: performance indicators and some analytical approaches", J. Urban Plann. Dev., vol. 128, no. 1, pp. 184-209, 2002.

[29] D. Greene, and M.Wegener, "Sustainable transport", J. Trans Geogr., vol. 5, no. 3, vol. 177-190, 1997.

[30] Y. Duvarci, and T. Yigitcanlar, "Integrated modelling approach for the transportation disadvantaged", J. Urban Plann. Dev., vol. 133, no. 3, pp. 188-200, 2007.

[31] J. Schmocker, M. Quddus, R. Noland, and M. Bell, "Estimating trip generation of elderly and disabled people", Transp. Res. Board, vol. 1924, no. 1, pp. 9-18, 2005.

[32] D. Ory, and P. Mokhtarian, "When is getting there half the fun?", Transport. Res. A, vol. 39, no. 1, pp. 97-123, 2005.

[33] D. Denmark, "The outsiders: planning and transport disadvantage", J. Plann. Educ. and Res., vol. 17, no. 3, pp. 231-240, 1998.

[34] R. Cervero, and K. Kockelman, "Travel demand and the 3Ds: density, diversity, and design", Transp. Res. Part D, vol. 2, no. 3, pp. 199-219, 1997.

[35] S. Schonfelder, and K. Axhausen, "Activity spaces", Transp. Policy, vol. 10, no. 1, pp. 273-286, 2003.

[36] K. Lucas, Ed, Running on empty: transport, social exclusion and environmental justice: Policy Press: Bristol 2004

[37] G. Currie, "Gap analysis of public transport needs: measuring spatial distribution of public transport needs and identifying gaps in the quality of public transport provision”, Transp. Res. Record, vol. 1402, no. 1, pp. 137-146, 2004.

[38] W. Anderson, "Urban form, energy and the environment: a review of issues, evidence and policy", Urban Stud., vol. 33, no. 1, pp. 736, 1996.
[39] D. Stead, and S. Marshall, "The relationships between urban form and travel patterns. An international review and evaluation", Eur J. Trans. Infrastruct. Res., vol. 1, no. 2, pp. 113-141, 2001.

[40] D. Stead, "Relationships between land use, socioeconomic factors, and travel patterns in Britain", Environ. Plann. B, vol. 28, no. 4, pp. 499-528, 2001.

[41] R. Kitamura, P. Mokhtarian, and L. Daidet, "A micro-analysis of land use and travel in five neighbourhoods in the San Francisco Bay Area", Transportation, vol. 24, no. 2, pp. 125-158, 1997.

[42] J. Holtzclaw, R. Clear, H. Dittmar, D. Goldstein, and P. Haas, "Location efficiency: neighbourhood and socio-economic characteristics determine auto ownership and use-studies in Chicago, Los Angeles and San Francisco", Transp. Plann. Technol., vol. 25, no. 1, pp. 1-27, 2002.

[43] H. Meurs, and R. Haaijer, "Spatial structure and mobility", Transp. Res. Part D, vol. 6, no. 6, pp. 429-446, 2001.

[44] Y. Susilo, and K. Maat, "The influence of built environment to the trends in commuting journeys in the Netherlands", Transportation, vol. 34, no. 5, pp. 589-609, 2007.

[45] F. Dieleman, M. Dijst, and G. Burghouwt, "Urban form and travel behaviour: micro-level household attributes and residential context”, Urban Stud., vol. 39, no. 3, pp. 507-527, 2002.

[46] P. Naess, "Accessibility, activity participation and location of activities: exploring the links between residential location and travel behaviour", Urban Stud., vol. 43, no. 3, pp. 627-652, 2006.

[47] R. Crane, and R. Crepeau, "Does neighbourhood design influence travel? A behavioural analysis of travel diary and GIS data", Transp. Res. Part D, vol. 3, no. 4, pp. 225-238, 1998.

[48] R. Cervero, and R. Gorham, "Commuting in transit versus automobile neighbourhoods", J. Am Plann. Assoc., vol. 61, no. 2, pp. 210-225, 1995 .

[49] J. Hine, and M. Grieco, "Scatters and clusters in time and space: implications for delivering integrated and inclusive transport", Trans. J., vol. 10, no. 1, pp. 299-306, 2003.

(C) Yigitcanlar et al.; Licensee Bentham Open.

This is an open access article licensed under the terms of the Creative Commons Attribution Non-Commercial License (http://creativecommons.org/licenses/by$\mathrm{nc} / 3.0 /$ ) which permits unrestricted, non-commercial use, distribution and reproduction in any medium, provided the work is properly cited. 